\title{
PRODUCT ROBUST DESIGN WITH A MIXTURE OF RANDOM AND INTERVAL FACTORS
}

\author{
Chuntao Liu, Zhihang Lin \\ CIM Institute, State Key Laboratory for Manufacturing Systems Engineering, Xi'an Jiaotong \\ University, China; Email: chtliu@mailst.xitu.edu.cn.
}

\begin{abstract}
To solve the product robust design problems involving a mixture of random and interval factors, a novel approach is proposed which combines an integrated metamodeling method with a percentile-based robust optimization model. The integrated metamodeling method embodies the integration of Response Surface Methodology (RSM) and Support Vector Regression (SVR), which is used to construct the metamodels of product performance efficiently. The percentile-based robust optimization model could bring both the design objective robustness and the feasibility robustness of the design constraint into account, which assures a product's reliability and quality robustness to the noise. A case study of a diaphragm spring in automobile clutch is described to show the effectiveness and practicability of the proposed approach.
\end{abstract}

Key words: robust design, random factors, interval factors, Response Surface Methodology, Support Vector Regression, percentile.

\section{INTRODUCTION}

Robust design is a method for improving the quality of a product through minimizing the effect of the causes of variation without eliminating the causes ${ }^{1}$. Under the concept of robust design, there are two broad categories of the causes of product performance variations: noise factors and control factors. In traditional robust design ${ }^{2}$, uncertainties associated with noise factors and control factors usually imply for randomness and nondeterministic factors are assumed to follow certain distributions. However, in reality, some of the distributions related to random factors are often not precisely known. Moreover, uncertainties associated with other uncertain factors may not be the result of randomness ${ }^{3}$. Therefore, when

This project is supported by Chinese National Natural High-Tech. R\&D Program for CIMS under Grant No. 2002AA413520 and National Fundamental Research Program (973) under Grant No. 2003CB716207.

Please use the following format when citing this chapter:

Liu, Chuntao, Lin, Zhihang, 2006, in International Federation for Information Processing (IFIP), Volume 207, Knowledge Enterprise: Intelligent Strategies In Product Design, Manufacturing, and Management, eds. K. Wang, Kovacs G., Wozny M., Fang M., (Boston: Springer), pp. 198-204. 
nondeterministic factors are only known within intervals, robust design approach should treat them as interval factors. So far, there is still no method to deal with the robust design problems involving the combination of random and interval factors.

The purpose of this paper is to describe an efficient method for robust design to deal with problems under the mixture of random and interval factors. In the proposed approach, an integrated metamodeling method based on Response Surface Methodology (RSM) and Support Vector Regression (SVR) is combined with a percentile-based robust optimization model in which both robustness of the design objective and feasibility robustness of the design constraint are simultaneously assured.

\section{A THREE-STEP ROBUST DESIGN APPROACH}

A three-step robust design approach is proposed (see Figure 1) to deal with the problems under the mixture of random and interval factors:

Step 1 Build approximation models to associate each product performance with control and noise factors by using an integrated metamodeling method based on RSM and SVR.

Step 2 Model the robustness of the design objective and feasibility robustness of the design constraint by using percentile-based formulations.

Step 3 Use a percentile-based robust optimization model to find the robust design solution.

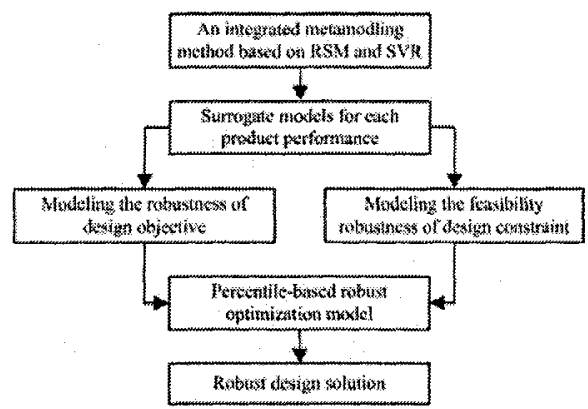

Figure 1. Flowchart of the three-step robust design approach

\subsection{Integration of RSM and SVR}

To take advantages of $\mathrm{RSM}^{4}$ and $\mathrm{SVR}^{5}$ and overcome their limitations, an integration strategy (as shown in Figure 2) is presented to build efficient surrogate models of product performance. 
This strategy first employs the RSM techniques to generate a number of design experiments and create response surface models to represent the performance behaviors under study. If the models are not accurate enough, the SVR technique is implemented. Here, support vectors are trained on a set of data points which correspond to the design experiments generated previously based on RSM techniques. Additional points would be added to achieve the desired accuracy. When the results from either RSM or SVR techniques are accurate enough, they can be used as approximation models in robust optimization programs to determine the optimal values.

With the integration strategy, it is anticipated that the limitation of RSM in modeling highly nonlinear behaviors could be overcome by the SVR techniques. In the situation when the Response Surface Model is accurate enough, the complicated training process of the SVR can be avoided.

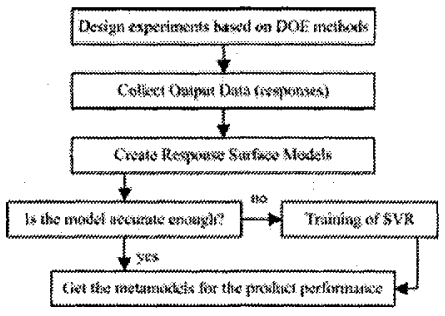

Figure 2. Flowchart of the integrated metamodeling method

\subsection{Percentile-based robust optimization model}

Under the mixture of random and interval factors, the percentile-based formulations are presented to model the robustness of design objective and the feasibility robustness of design constraint.

1) Modeling the robustness of design objective

Under the mixture of random and interval factors, the control factors $\boldsymbol{x}$ and noise factors $\boldsymbol{z}$ are classified into two types of factors: random factors $\boldsymbol{r}$ characterized by probability distributions; interval factors $d$ within specified intervals $\left[\boldsymbol{d}_{L}, \boldsymbol{d}_{U}\right]$. As a result, the design objective $y=y(\boldsymbol{x}, \boldsymbol{z})=y(\boldsymbol{d}, \boldsymbol{r})$ is characterized by the mixture of distribution and interval as well. $y \mid \boldsymbol{d}$ is a random variable which is the function of random variables $\boldsymbol{r}$ and can be described by a marginal distribution, as $d$ varies within the interval $\left[\boldsymbol{d}_{L}, \boldsymbol{d}_{U}\right], y$ performs as a sequence of probability distributions In this distribution sequence, there exists a maximum $y^{p_{2}}$, denoted as $y_{\max }^{p_{2}}$, and a minimum $y^{p_{1}}$, denoted as $y_{\min }^{p_{1}}$, where $p_{1}$ is a left-tail cumulative distribution functions (CDF), for example, 0.05 or 0.1 , representing the product performance at the 
left tail of its probability distribution and $y^{p_{2}}$ is a right-tail CDF, for example, 0.95 or 0.99 . The percentile difference $\left.\Delta y_{p_{1}}^{p_{2}}\right|_{\max }=y_{\max }^{p_{2}}-y_{\min }^{p_{1}}$ reflects the variation range of a design objective. $\overline{y^{0.5}}$ is used to represent the variation location of a design objective.

Under the mixture of random and interval factors, for smaller-the-better type robust design, the robustness of design objective can be modeled as

$$
\operatorname{minimize}\left[\overline{y^{0.5}},\left.\Delta y_{p_{1}}^{p_{2}}\right|_{\max }\right]
$$

2) Modeling the feasibility robustness of design constraint

Similarly, under the mixture of random factors $r$ and interval factors $d$, the constraint function $g_{j}(x, z)=g_{j}(d, r)$ performs as a sequence of probability distributions. The feasibility robustness of design constraint under the mixture of random and noise factors can be modeled as

$$
\left.g_{j}^{p_{j}}\right|_{\max } \leq 0 \quad j=1,2, \cdots, m
$$

Where $p_{j}$ is the user specified reliability.

In summary, through using the concept of percentile, under the mixture of random and interval factors, the unified robust optimization model for integrated design objective robustness and feasibility robustness of design constraint can be given as

$$
\begin{array}{ll}
\min & \left.\overline{\left[y^{0.5}\right.},\left.\Delta y_{p_{1}}^{p_{2}}\right|_{\max }\right] \\
\text { s.t. } & \left.g_{j}^{p_{1}}\right|_{\max } \leq 0 \quad j=1,2, \cdots, m \\
& \boldsymbol{x}_{L} \leq \overline{\boldsymbol{x}} \leq \boldsymbol{x}_{U}
\end{array}
$$

\section{A CASE STUDY}

The design of a diaphragm spring in automobile clutch is used as a case study to verify the approach described in this paper. The purpose of this design is to find the dimensions of the spring as shown in Figure 3, which can meet the following design requirements:

1) Minimize the maximum stress $\sigma$ in the diaphragm spring.

2) Produce sufficient load when the diaphragm spring clamps the pressure plate. 


$$
g_{1}=4 T_{\max }\left(D^{2}-d^{2}\right) /\left(D^{3}-d^{3}\right)-P\left(\lambda_{b}\right) \leq 0
$$
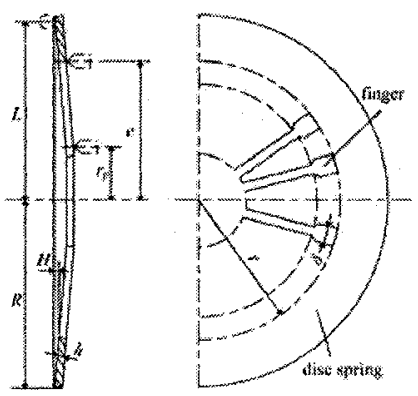

Figure 3. A diaphragm spring

3) Transmit the engine's power stably even when the clutch disc is worn.

$$
g_{2}=\left|P\left(\lambda_{b}-1.2\right)-P\left(\lambda_{b}\right)\right|-0.05 P\left(\lambda_{b}\right) \leq 0
$$

Factors that are related to the requirements above are classified into eight control factors and three noise factors. In this case, the control factors are random variables and noise factors are interval variables (listed in Table 1).

Table 1. Information of random and interval factors

\begin{tabular}{ccc}
\hline \multicolumn{3}{c}{ Random factors } \\
\hline Factor & Standard deviation & Distribution \\
\hline$R / \mathrm{mm}$ & 0.04 & Normal \\
$r / \mathrm{mm}$ & 0.04 & Normal \\
$H / \mathrm{mm}$ & 0.05 & Normal \\
$h / \mathrm{mm}$ & 0.05 & Normal \\
$L / \mathrm{mm}$ & 0.02 & Normal \\
$e / \mathrm{mm}$ & 0.02 & Normal \\
$r_{p} / \mathrm{mm}$ & 0.02 & Normal \\
$\delta / \mathrm{mm}$ & 0.02 & Normal \\
\hline \multicolumn{3}{|c}{} \\
\hline Factor & Interval factors \\
\hline$T_{\max } / \mathrm{N} \cdot \mathrm{m}$ & Lower Bound & Upper Bound \\
$D / \mathrm{mm}$ & 178.2 & 192.2 \\
$d / \mathrm{mm}$ & 239.6 & 240.4 \\
\hline
\end{tabular}

In this design problem, $\sigma$ is treated as the design objective, while $g_{1}$ and $g_{2}$ are treated as the design constraint. To relieve the computational burden, the approximate models of $\sigma, g_{1}$ and $g_{2}$ are constructed by using the integrated metamodeling method aforementioned. Three metrics, named as, Max. Absolute Error (MAE), Average Absolute Error (AAE) and Root 
Mean Square Error (RMSE) are used to measure the accuracy of metamodels. To be as a contrast, Artificial Neural Networks (ANN) models for the product performance on the same sample data are also built and tested. The accuracy of RSM, SVR and ANN models is shown in Table 2.

It could be noted from Table 2 that, for $\sigma$, which is highly nonlinear, the accuracy of SVR is the best while ANN is better than RSM. For $g_{1}$ and $g_{2}$, which are also nonlinear but not as much as $\sigma$, three methods have achieved a good accuracy - the accuracy of RSM is close to that of SVR while ANN is the worst. This indicates that, in most cases, SVR is better than RSM and ANN. The limitations of RSM and ANN, i.e., the defect of RSM in modeling highly nonlinear behaviors, and the necessity of a large set of training data for ANN, could be eliminated by SVR techniques. In the case of modeling lowly nonlinear behaviors, the use of RSM makes the complicated training process of SVR and ANN avoided.

Table 2. Accuracy of metamodels

\begin{tabular}{lccccccccc}
\hline \multirow{2}{*}{ Error } & \multicolumn{3}{c}{ RSM } & \multicolumn{4}{c}{ SVR } & \multicolumn{3}{c}{ ANN } \\
\cline { 2 - 10 } & $\sigma$ & $g_{1}$ & $g_{2}$ & $\sigma$ & $g_{1}$ & $g_{2}$ & $\sigma$ & $g_{1}$ & $g_{2}$ \\
\hline MAE & 2.739 & 0.485 & 0.178 & 0.524 & 0.473 & 0.165 & 1.724 & 0.959 & 0.175 \\
AAE & 0.457 & 0.098 & 0.029 & 0.073 & 0.095 & 0.026 & 0.287 & 0.161 & 0.028 \\
RMSE & 0.965 & 0.199 & 0.061 & 0.145 & 0.193 & 0.055 & 0.586 & 0.342 & 0.059 \\
\hline
\end{tabular}

After constructing the metamodels of the design objective and design constraint, the percentile-based robust optimization model is given as

$$
\begin{array}{ll}
\min & {\left[\overline{\sigma^{0.5}},\left.\Delta \sigma_{0.01}^{0.99}\right|_{\max }\right]} \\
\text { s.t. } & \left.g_{j}^{0.99}\right|_{\max } \leq 0 \quad j=1,2
\end{array}
$$

Compromise Programming method ${ }^{6}$ is used to solve this multi-objective model which allows the designer assign a weight factor $w$ representing the relative importance of the two objectives. The optimal solutions under three different values of the weight factor are listed in Table 3.

For comparison, we also solve the problem by using the traditional deterministic optimization model ignoring the uncertainty in control and noise factors. The optimal solution is given in Table 4. To compare the effectiveness of the different methods, the variation range of stress, $\left.\Delta \sigma_{0.01}^{0.99}\right|_{\max }$, and the probability of constraint satisfaction at the optimum point from the conventional deterministic model are also calculated.

It can be noted from Table 3 and Table 4, the variation range of stress of the robust design solution is much less than that of the traditional deterministic optimization model, which means that the robust design 
solution is less sensitive to the variations in design conditions or operating conditions. Meanwhile, as predicted, the conventional deterministic model generates a low probability for constraint satisfaction, while the robust design solution produces the satisfying constraint feasibility.

Table 3. Robust design solutions of the diaphragm spring design

\begin{tabular}{ccccccccccc}
\hline$w$ & $\begin{array}{c}R \\
/ \mathrm{mm}\end{array}$ & $\begin{array}{c}r \\
/ \mathrm{mm}\end{array}$ & $\begin{array}{c}H \\
/ \mathrm{mm}\end{array}$ & $\begin{array}{c}h \\
/ \mathrm{mm}\end{array}$ & $\begin{array}{c}L \\
/ \mathrm{mm}\end{array}$ & $\begin{array}{c}e \\
/ \mathrm{mm}\end{array}$ & $\begin{array}{c}r_{\mathrm{p}} \\
/ \mathrm{mm}\end{array}$ & $\begin{array}{c}\delta \\
/ \mathrm{mm}\end{array}$ & $\begin{array}{c}\overline{\sigma^{0.5}} \\
/ \mathrm{MPa}\end{array}$ & $\begin{array}{c}\left.\Delta \sigma_{0.01}^{0.99}\right|_{\max } \\
/ \mathrm{MPa}\end{array}$ \\
\hline 0.3 & 113 & 85 & 4.29 & 2.67 & 110 & 89.0 & 31 & 10 & 1439 & 21.01 \\
0.5 & 116 & 90.1 & 4.32 & 2.70 & 112 & 92.4 & 30 & 9 & 1384 & 26.61 \\
0.7 & 121 & 95.8 & 4.38 & 2.77 & 119 & 98.7 & 32 & 9 & 1341 & 33.34 \\
\hline
\end{tabular}

Table 4. Optimal solution from the deterministic optimization method

\begin{tabular}{ccccccccccc}
\hline$R$ & $r$ & $H$ & $h$ & $L$ & $e$ & $r_{\mathrm{p}}$ & $\delta$ & $\left.\Delta \sigma_{0.01}^{0.99}\right|_{\max }$ & \multicolumn{2}{c}{$\begin{array}{c}\text { Probability of } \\
\text { constraint } \\
\text { satisfaction }\end{array}$} \\
\hline$/ \mathrm{mm}$ & $/ \mathrm{mm}$ & $/ \mathrm{mm}$ & $/ \mathrm{mm}$ & $/ \mathrm{mm}$ & $/ \mathrm{mm}$ & $/ \mathrm{mm}$ & $/ \mathrm{mm}$ & $/ \mathrm{MPa}$ & $g_{1}$ & $g_{2}$ \\
\hline 123 & 100 & 4.19 & 2.66 & 121 & 103 & 30 & 11 & 61.21 & 0.58 & 0.791 \\
\hline
\end{tabular}

\section{CONCLUSION}

When both the random factors and interval factors present in a product robust design problem, the product performance is also characterized with the mixture of randomness and interval. An integrated metamodeling method is combined with a percentile-based robust optimization model to solve this problem. The future work will target at how to improve the efficiency of solving the optimization model.

\section{REFERENCES}

1. M. S. Phadke, Quality Engineering Using Robust Design (Prentice Hall, New Jersey, 1989).

2. W. Chen, J. K. Allen, F. Mistree, and K. L. Tsui, A procedure for robust design, ASME Journal of Mechanical Design 118 (4), 478-485 (1996).

3. X. Du, A. Sudjianto, and B. Huang, Reliability-based design under the mixture of random and interval variables, ASME Journal of Mechanical Design 127(6), 1068-1076 (2005).

4. R. H. Myers, and D. C. Montgomery, Response Surface Methodology: Process and Product Optimization Using Designed Experiments (John Wiley and Sons, New York, 1995).

5. N. Cristianni, and J. Shawe-Taylor, An introduction to support vector machines and other kernel-based learning methods (Cambridge University Press, Cambridge, 2000).

6. M. Zeleny, Compromise programming, in Multiple Criteria Decision Making (University of South Carolina Press, Columbia, 1973). 